\title{
¿CREACIÓN O EVOLUCIÓN? EL ETERNO DEBATE ACERCA DE LA EXISTENCIA
}

\section{¿CREATION OR EVOLUTION? THE ETERNAL DEBATE ABOUT EXISTENCE}

Referencia del ensayo

Paiz Varela, R. (2022). ¿Creación o evolución? el eterno debate acerca de la existencia. Revista Científica del Sistema de Estudios de Postgrado de la Universidad de San Carlos de Guatemala, 5(1), 39-45. DOI: https://doi.org/10.36958/sep.v5i1.95

\author{
Rocael Paiz Varela \\ rpaiz@fahusac.edu.gt \\ https://orcid.org/0000-0003-0528-4869 \\ Maestría en Filosofía \\ Universidad de San Carlos de Guatemala
}

Fecha de aceptado: 05/01/2022

\section{ABSTRACT}

OBJECTIVE: to carry out an objective and impartial analysis of the two positions defined in the course of history, which has led to debates and disputes, with the intention that the use of reason, discernment, analysis and philosophical reflection prevail. METHOD: the descriptive and hermeneutical methodology was used, revealing the ideas, criteria, thoughts and commenting on opinions established by various ancient, modern and contemporary thinkers and philosophers. RESULTS: it has been possible to establish that the two theories mentioned about the origin of existence continue to be the object of discussion and debate because the scientific world does not accept the creationist theory and the religious world does not accept evolution either, and in these positions we human beings find ourselves at a crossroads because according to beliefs, traditions, religion and study we have the need to prefer one of them. CONCLUSION: although the two positions or theories about the origin of existence have been debated for many years, the fact of trying to reconcile them becomes increasingly impossible. In this frivolous and materialistic world in which we live, it seems that the new generations are not attracted to the debate, this added to the superficial and indifferent attitude that the majority shows, sadly indicates that they are not interested in discussing or investigating the cause of our existential origin.

\section{KEYWORDS}

Science, religion, god, reason, existence 


\section{INTRODUCCIÓN}

¿Cómo se originó la vida, cómo apareció el hombre y cómo es que existimos en este planeta al que se le dio el nombre de Tierra? ¿Existimos por creación divina o por medio de ese proceso llamado evolución?, ¿Somos, según la biblia, criaturas o seres creados a la imagen y a la semejanza de Jehová?, o ¿es nuestra vida o nuestra existencia un proceso que ha tardado muchos años y que nos ha llevado a lo que somos en la actualidad?

Plantear estas interrogantes y encontrar una respuesta que pueda satisfacer a todos se vuelve una tarea sumamente difícil ante la multiplicidad de criterios, opiniones y creencias que son parte de la religiosidad y del pensamiento filosófico de hombres y mujeres alrededor del mundo entero y durante todas las épocas en que al ser humano le ha tocado vivir.

A pesar de la existencia de otras teorías acerca del origen de la vida hoy nos vamos a referir a la controversia o debate que se ha dado desde hace muchos años entre el creacionismo y el evolucionismo y que se ha convertido en una eterna disputa acerca de la existencia.

¿Es la vida o la existencia fruto de la creación o un proceso evolutivo? Durante muchos años, los seres humanos hemos sido testigos de la controversia y diversidad de criterios acerca de la pregunta inicial de este tema. Creyentes versus no creyentes o religiosos contra ateos han disputado y llevado una confrontación más allá de la reflexión, del análisis y la indagación, llegando, incluso, a enfrentamientos extremos. En nombre de la corriente cristiana; en nombre de un supuesto Dios; alzando la bandera de un ser supremo como hacedor de todo cuanto existe, en nombre de la negación de ese dios, de Jesús y la virgen María; en nombre del ateísmo más exagerado y violento y en nombre de los extremismos más radicales se han librado bata- llas, se han asesinado miles de personas y se han desaparecido pueblos.

Esto ha ido más allá del debate; se ha ido a la confrontación, a las guerras y hasta genocidios con el fin supremo y objeto fundamental de imponer un criterio, establecer una opinión, implantar una creencia y hasta obligar a profesar una religión o alejarse de ella.

\section{¿A qué se le ha dado el nombre de crea- ción?}

Por creación vamos a comprender a todo aquello que se puede crear, instaurar, inventar, que se da de manera original, que no estaba o no existía con anterioridad. Los hombres somos en el mundo los únicos seres pensantes, atrevidos y osados que poseemos la capacidad de idear cosas, fabricar objetos, elaborar teorías, escribir obras, dibujar pinturas, componer música, construir vehículos, planificar edificios, reconstruir la historia, hablar distintos idiomas, estudiarnos a nosotros mismos, reproducirnos, cultivar la tierra, dominar el espacio, inventar la tecnología, navegar sobre y debajo de los mares, imaginar y desarrollar, crear empresas y muchas cosas más.

A través de esto podríamos afirmar que la creación puede darse a partir de las ideas de individuos capaces de imaginar o concebir algo; establecerse metas, abrir negocios o legitimar cuestiones de orden laboral que puedan cumplir una función importante para resolver una problemática o bien para solventar una necesidad sea esta individual o de carácter colectivo.

En términos generales, la creación tiene que ver mucho con la idea de encontrar soluciones a diversas dificultades, a problemáticas existentes o responder a diversas dudas que se plantean en el transitar de la vida. 
El término «creación»» puede entenderse filosóficamente en cuatro sentidos: 1 . Producción humana de alguna cosa partiendo de una realidad que no existe, pero en tal forma que lo que se produce no se encuentre necesariamente en esa realidad; 2 . Producción natural de algo a partir de algo preexistente, pero sin que el efecto esté incluido en la causa, o sin que haya estricta necesidad de tal efecto; 3. Producción divina de una cosa partiendo de algo preexistente, lo que da como resultando un orden o un cosmos de un anterior desorden y 4. Producción divina de algo a partir de la nada o creatio ex nihilo (Ferrater, 1979).

También podría afirmarse que es un deseo del hombre que puede surgir por medio de su labor constante y puede utilizar recursos que tiene en su entorno y en su realidad.

Platón consideró a la creación como obsesión divina; Schelling creía que era algo así como un movimiento que partía de lo consciente a lo inconsciente; Von Hartman pensaba en un hálito vivificante de lo inconsciente; Bergson como una intuición mística; Freud como manifestación de los instintos (Diccionario filosófico, 1999).

\section{¿Qué es el creacionismo?}

Podríamos definir al creacionismo como una creencia religiosa nacida bajo el supuesto de que todo cuando existe, hombre y naturaleza, fueron creados por un ser supremo, poderoso, omnipotente o bien, por actos concretos de una divinidad, a la cual se le ha llamado Dios, Jehová, Yavé o Alá.

Es una idea basada en la religión y profesada por los creyentes en todo el mundo donde sustentan que la vida, y por ende los seres humanos, tienen su origen en algo divino.
También conocida o nombrada como teoría de la creación ha sido considerada como una doctrina religiosa que parte de la idea que el universo fue creado como un acto de voluntad consciente y concreto de una divinidad. Esta idea o esta creencia se encuentran sostenidas y fundamentadas en diversas religiones.

En la historia del mundo occidental este supuesto creacionismo, se fundamenta en los relatos de la creación que se encuentran descritos en la biblia y especialmente en su primer libro, el Génesis, donde se relata cómo un ser supremo creo todo en seis días y descanso al séptimo.

\section{¿Es la existencia el resultado de la crea- ción divina?}

Para la iglesia católica y todos los grupos, sectas o denominaciones religiosas nacidas después de que Martín Lutero en 1517 iniciara un movimiento de reforma protestante en el seno de la iglesia católica, la aparición de los seres humanos sobre la faz de la tierra se dio por medio de inspiración o voluntad divina y su explicación o relato se encuentra en la Biblia, libro sagrado tanto para la iglesia como para la corriente cristiana.

A través de la historia, la biblia ha recibido diferentes nombres, en consideración también a los idiomas y lenguas habladas en el mundo donde han llegado la influencia religiosa católica primero y distintas denominaciones religiosas después, por ejemplo: para los griegos la Biblia, anteriormente se conocía como Septuaguinta, ya que era la única versión que existía en ese idioma; para los hebreos y arameos recibe el nombre de Tanaj o Mikrá y también la Torá (el Pentateuco).

Para los musulmanes o para los seguidores del islam, su libro sagrado recibe el nombre 
de Corán donde se encuentra la palabra de Dios y se considera que la palabra fue revelada a Mahoma, su profeta.

Al leer o al interpretar el libro de Génesis, aparece que Dios creo ordenadamente y en una semana la materia y la luz, logrando que las especies animales se movieran con toda tranquilidad. También creo todos los medios necesarios para que los seres vivos tuvieran sustento natural, para que pudieran vivir, reproducirse y desarrollarse. Por último se dio a la tarea de crear al hombre con características diferentes y con facultades distintas a lo que había creado antes.

La idea de la creación divina se sustenta en dos pilares importantes: fe y revelación.

Para Santo Tomás, además del conocimiento del mundo, el hombre aspira, sobre todo, al conocimiento de Dios, al que podemos llegar por otras dos vías más, que son la fe y la revelación. Es decir, a Dios lo podemos conocer mediante la revelación, pero también por la razón.

\section{¿Qué es fe y qué es revelación?}

En todos los países existen grupos religiosos, sectas o denominaciones las que al final de todo, lo que prometen es que al momento de la muerte los seres humanos tendremos una vida eterna y que de algún modo nos vamos a encontrar nuevamente con nuestros seres queridos, amistades y familia. Esta propuesta logra en los creyentes un consuelo espiritual. Generalmente la religión promete la salvación por medio único de la fe. Si esta existe y si los seres humanos tenemos fe en Dios, este nos hará salvos.

La fe se ha considerado como un concepto de difícil definición. Se podría afirmar que es un término vital de la religión o del creyente que va referida al acto de creer ciegamente en algo, ya sea una deidad o en un conjunto propio de la religión o bien, cuando menos estar esperanzados de que algo sucederá como deseamos o la convicción de que algo será como lo queremos.

Es pues, la fe, la certeza de lo que se espera, la convicción de lo que no se ve (Hebreos 11:1 Biblia Latinoamericana 2005). Comprendemos la fe como el creer en algo, religiosamente en la existencia de un ser divino que es capaz de crear y de obrar milagros por su infinita bondad al cual se sigue de acuerdo a sus preceptos y a las doctrinas de la iglesia. Durante mucho tiempo se ha identificado a la fe con la creencia y a la creencia con la fe, pero una fe y una creencia opuestas al saber o que se oponen también al conocimiento.

Se ha comprobado que todo saber, todo conocimiento tiene en su base una creencia. Es claro entonces que, en la fe, la creencia ha suscitado en el curso de toda la Edad Media las distintas formas que guardan estrecha relación con el conocimiento.

Por su lado, la revelación va a aparecer en las ideas religiosas y casi siempre viene a referirse al obtener una afirmación que se mantenía oculta o de forma secreta y que era dada a individuos que tenían algunas facultades especiales por alguien sobrenatural. En algunos pasajes de la biblia se da a conocer que la cosa revelada se da entre Dios y los personajes llamado profetas.

En los grupos religiosos como el hinduismo la idea de revelación recibe el nombre de Struti, que viene a ser algo así como los textos revelados y a ello se opone el Smrti que son una cantidad de textos llamados tradicionales. Para el judaísmo, el cristianismo y el islam existe la revelación divina que fue dada especialmente a Moisés en las tablas de los 10 mandamientos. Ese concepto de revelación va a oponerse en la actualidad al con- 
cepto científico del conocimiento que se adquiere mediante el esfuerzo de razonar para poder, entender y comprender las cosas.

\section{El cambio y el tiempo}

Por cambio podríamos mencionar cuando un elemento pasa de un estado a otro, una transformación, un movimiento, un desplazamiento. Todo en la vida es un cambio.

Durante todo el transcurso de la historia del mundo y del hombre nos hemos dado cuenta o hemos sido capaces de observar que todo cambia, todas las cosas, los objetos y nosotros mismos estamos cambiando. No somos los mismos de ayer ni seremos los mismos de mañana, todo es un cambio. En la vida y en las cosas que nos rodean no existe lo estático. En el transcurrir del tiempo, meses y años, especialmente, podemos ser testigos directos que todo aquello que nos rodea se va transformando y por lo tanto va cambiando. Parménides, filósofo griego afirmaba que el cambio permanente y continuo es lo único que no puede cambiar. Está presente en todo y es el todo. Todo cambia, se transforma, se modifica.

Uno de los grandes filósofos griegos, Heráclito de Efeso, en un momento determinado de su quehacer filosófico acuñó la frase: "nadie se baña dos veces en un mismo río", de eso podemos concluir en que todo cambia en el río y en el que se baña, porque todo cambia y se transforma en la realidad.

Cambio también es sinónimo de mutación. El mutacionismo tiene su fundamento en la explicación de la transformación de especies donde irrumpen y se realizan pequeños cambios producidos al azar en el transcurrir de varias generaciones.

El tiempo es sinónimo de cambio y para Platón, ese tiempo es como una imagen que se mueve por la eternidad. El número del movimiento con respecto al antes y al después lo era para Aristóteles; Plotino toma en consideración a la vida del alma en movimiento al pasar de un estado de acción o experiencia a otro.

\section{La evolución}

Podemos definirla como un cambio, una transformación gradual y a través del tiempo especialmente de los seres vivos que, a través de ese tiempo y espacio, vienen sufriendo transformaciones sustanciales que los diferencian de épocas y tiempos pasados. Se cree que este proceso ha originado la diversidad de formas de vida que existen sobre la Tierra a partir de un antepasado común.

Evolución proviene de evolutio, del verbo evolvo, el vocablo 'evolución' designa la acción y efecto de desenvolverse, desplegarse, desarrollarse algo. La palabra conserva todavía su sentido genérico de desarrollo, pero a menudo se la usa para designar una doctrina particular que se denomina como una teoría.

¿Es la existencia el resultado de la evolución?

Para los evolucionistas el punto central de su pensamiento radica en la premisa que la vida evolucionó sobre la faz de la tierra. De formas pequeñas y sin importancia se derivan formas más complejas y todas comparten antecesores comunes.

Hace más o menos unos 162 años el mundo de la ciencia sufrió un importante cambio ya que un estudioso, investigador y naturalista inglés de apellido Darwin y de nombre Charles, de acuerdo a estudios e investigaciones realizadas, le llevaron a publicar una de las obras más importantes del mundo científico de su época titulada: "El Origen de las Especies" como resultado de varios años de años 
de observaciones, viajes y visitas a distintas partes del mundo. En ese documento Darwin afirmó que de un antepasado común se originaron todas las especies que existen en la Tierra; que las aves, los peces, las flores y nosotros, los humanos así como también los vegetales, estamos relacionados, venimos de lo mismo, lo que significa que todos tenemos un origen común, algo de lo que venimos y por lo que somos en la actualidad.

Darwin creía firmemente que las especies habían llegado a evolucionar debido a un proceso al cual le dio el nombre de "selección natural", que ha consistido en una adaptación con gran éxito de las especies que eran más fuertes a las necesidades cambiantes del lugar que habitaban y que las especies débiles que pudieran existir, que no pudieran adaptarse a los cambios por venir no se reproducían y por lo tanto, no podrían sobrevivir. Según esa selección natural darwiniana se puede causar a lo largo de muchas generaciones los cambios en una especie, en su color o también en su tamaño. Este proceso ha venido a definirse como microevolución. Pero esta selección natural es capaz también de crear otras especies que no existían, lo que se conoce como macroevolución.

Históricamente, la idea de que la vida en la tierra evolucionó a partir de un tronco o un ancestro común ya había sido formulado en la filosofía griega. La idea que afirma que los seres vivos o las especies se fueron transformando con el tiempo y de forma contínua fue argumentada por muchos científicos de los siglos XVIII y XIX, a los cuales Darwin citó en el primer capítulo de su libro El origen de las especies. Algunos filósofos griegos antiguos contemplaron la posibilidad de cambios en los organismos a través del tiempo (Mayr, 1982).

Alrededor de mil años antes de que lo hiciera Darwin, un filósofo musulmán que vivía en Irak, conocido como al-Jahiz, escribió un libro sobre cómo los animales cambian a través de un proceso que él llamó "selección natural" (BBC News, 2019).

\section{CONCLUSIÓN}

Podemos definir entonces que tanto la idea de creación a través de un acto inspirado por un ser supremo como la evolución, cambio y transformación del ser humano en forma evolutiva, no son ni postulados ni teorías nuevas. Estas dos posiciones antagónicas en el transcurso de la historia llevan formulándose hace muchos, muchos años. La idea de la existencia por medio de la creación está fundamentada en la religión y, principalmente, en la fe y en la revelación.

La idea de la evolución se encuentra basada en la ciencia y en determinados momentos se han presentado pruebas de que la existencia se ha dado por los cambios y las transformaciones de los seres vivos a través de miles de años, pruebas aceptadas por el mundo científico, más no para el mundo religioso. No se puede dejar a un lado, ni se puede excluir una de estas teorías. Será el ser humano el que, por medio de su conciencia, sus creencias, su religión, sus estudios y su pensamiento científico pueda determinar, para él, aquello que crea exacto, serio y verdadero.

No es posible aceptar nunca las posturas adoptadas por seguidores de ambas concepciones que asumen actitudes violentas para hacer valer su criterio. Se debe debatir con análisis y argumentos serios, con respeto y con tolerancia a los pensamientos de otras personas para llegar a un punto de convergencia, aunque esto sea una tarea harto difícil.

Darwin no fue el creador ni el inventor de la "selección natural". Este concepto ya había sido formulado cientos de años antes por un filósofo musulmán. El pensamiento y la re- 
flexión filosófica no puede dejar a un lado ni tomar partido por una de las posiciones, sino aceptar estas y otras que también existen y fomentar el diálogo y el análisis serio, imparcial y objetivo mediando el uso de la reflexión y por, sobre todo, imperando siempre el camino de la razón.

\section{REFERENCIAS}

BBC News. (2019). Al-Jahiz, el filósofo musulmán que tuvo la idea de la evolución 1.000 años antes que Darwin. Obtenido de BBC New Mundo: https://www.bbc.com/ mundo/noticias-47477067\#: :text=Alrededor\%20de\%20mil\%20a\%C3\%B1os\%20antes,\%C3\%A91\%20llam\%C3\%B3\%20\%22selecci\%C3\%B3n\%20natural\%22

Biblia Latinoamericana. (2005). Editorial Verbo Divino.
Ferrater, J. (1979). Diccionario de filosofía (Sexta ed.). Alianza Editorial. https:// www.diccionariodefilosofia.es/es/buscador.html?search=CREACl\%C3\%93N+.

Mayr, E. (1982). El crecimiento del pensamiento biológico: diversidad, evolución y herencia. Universidad de Cambridge. https://www. Dialnet-ErnstMayr19042005-2327029.pdf.

\section{SOBRE EL AUTOR}

\section{Rocael Paiz Varela}

Es educador, poeta, escritor y periodista, $\mathrm{Br}$. Industrial y Perito en una especialidad, PEM en Pedagogía y Ciencias de la Educación, Lic. en Pedagogía y Ciencias de la Educación, Licenciado en Pedagogía y Administración Educativa, Maestro en Filosofía. Egresado de la Escuela de estudios de Posgrado de la USAC. Profesor Titular de la Facultad de Humanidades de la Universidad de San Carlos de Guatemala.

\author{
Conflicto de intereses \\ Declara no tener ningún conflicto de intereses. \\ Declaración de consentimiento informado \\ El estudio se realizó, respetando el Código de ética y buenas prácticas editoriales de publicación. \\ Derechos de uso \\ Copyright $\odot 2022$ por Rocael Paiz Varela \\ Este texto está protegido por una licencia Creative Commons 4.0. Internacional
}

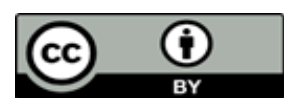

Usted es libre para compartir, copiar y redistribuir el material en cualquier medio o formato y adaptar el documento, remezclar, transformar y crear a partir del material para cualquier propósito, incluso comercialmente, siempre que cumpla la condición de atribución: usted debe reconocer el crédito de una obra de manera adecuada, proporcionar un enlace a la licencia, e indicar si se han realizado cambios. Puede hacerlo en cualquier forma razonable, pero no de forma tal que sugiera que tiene el apoyo del licenciante o lo recibe por el uso que hace. 